\title{
Nonconservation of energy by the vacuum
}

\author{
Gregor W. Bayer ${ }^{\text {a }}$ \\ a 1542 Tuley Street, Cedar Hill, Texas 75104, USA
}

\begin{abstract}
If collapsed objects or black holes absorb energy from the vacuum, then their increasing mass will generate a repulsive gravitational force, which might account for the present cosmic acceleration. Direct evidence for vacuum instability should be sought in high-energy particle collisions, where energy conservation has not been seriously tested.
\end{abstract}

Key words: gravitation, cosmology:theory, black hole physics, elementary particles PACS: 98.80.-k, 98.80.Cq, 04.40.Nr, 47.75.+f

\section{Introduction}

Thanks to the failed efforts of countless perpetual-motion-machine inventors we can be confident that energy is conserved, at least in those situations where it has been thoroughly tested. Most common processes are well described by quantum electrodynamics and Newtonian gravitation, whose equations of motion conserve a number called the energy. So useful is this concept that energy is assumed to be conserved even when the exact equations of motion are unknown, such as in high-energy collisions. However, there are reasons to suspect that energy is not always conserved.

(1) Ultra-high-energy cosmic rays [Cronin (1999)] present us with two major puzzles: How do particles get accelerated to such high energies, and how can they penetrate very far through the cosmic microwave background without being stopped by interactions? Maybe they are not as energetic as we think, because energy is created in the atmospheric collision event.

(2) Energy conservation is not a direct consequence of Poincaré symmetry; it also involves an assumption about the stability of the vacuum, which may not hold true in extreme situations.

Email address: gbay@att.net (Gregor W. Bayer). 
(3) In an expanding Universe, there must be matter creation in one form or another. The Steady-state Theory [Kragh (1996)], [Hoyle et al. (2000)] proposes that matter is continuously being created so as to maintain a constant density as the Universe expands. Whereas in the Big Bang Theory, all matter was created suddenly, and then production stopped altogether. A closed, cyclic Universe, where the total mass-energy remains constant, appears to be ruled out by the data.

(4) It is believed that the vacuum plays an important role in the determination of mass. The Higgs mechanism of spontaneous symmetry breaking shifts the Lagrangian by a constant amount. This has no effect so far as the electroweak interactions are concerned, but it is of great consequence if gravitation is included. Energy nonconservation, if it occurs at all, would most likely be found in the production of Higgs bosons, which would clearly indicate the presence of false vacuum. Below the Higgs threshold, say, the vacuum is likely to be perfectly stable.

(5) The same physical process that gave rise to the Big Bang may now be taking place within collapsed objects or black holes, where extreme conditions must occur. A slow accretion of energy from the vacuum would cause the total energy of the Universe to increase, thereby driving the present cosmic acceleration [Riess et al. (1998)], [Perlmutter et al. (1999)].

\section{Gravitational Repulsion}

Whenever new mass-energy is created, it must be accompanied by a repulsive gravitational force. To show this, let us begin by writing the energy-momentum tensor in the general fluid form

$$
T_{\mu \nu}=(\rho+\lambda) u_{\mu} u_{\nu}+(p-\eta-\lambda)\left(u_{\mu} u_{\nu}-g_{\mu \nu}\right) .
$$

Here, $\rho$ is the mass-energy density, $p$ is the material pressure, $\lambda=\Lambda / 8 \pi G$, and $\Lambda$ is the cosmological constant. Also included is a non-standard property, the creativity $\eta$, which acts like a negative pressure, and is normally absent. Since Bronstein (1933) [Kragh (1996)], much has been written about matter creation, and creativity has been extensively discussed in the literature [Prigogine et al. (1989)], [Lima et al. (1996)], where various derivations and justifications are given. Other names for creativity: creation pressure, generalized bulk viscosity, etc.

Since the energy-momentum tensor is proportional to a geometrical quantity, which has zero divergence, it must satisfy $T_{; \nu}^{\mu \nu}=0$. In a Robertson-Walker universe, the time component of this condition is the material energy equation

$$
\dot{M}=\dot{V}(\eta-p), \quad M=\rho V, \quad V=(4 \pi / 3) a^{3} .
$$


The factor $4 \pi / 3$ has no effect because it cancels out of the equations. Using the equation of state $p=w \rho$, the material energy is defined by

$$
\frac{d E}{d V}=\frac{d\left(V^{w} M\right)}{V^{w} d V}=\eta
$$

Thus, for $\eta=0$, we obtain $M \propto V^{-w}$.

Meanwhile, the Einstein field equation gives the gravitational energy equation

$$
\frac{\dot{a}^{2}+k}{2 a^{2}}-\frac{4 \pi G}{3}(\lambda+\rho)=0
$$

where $a$ is the scale factor, and $k$ is the curvature. This simply says that the cosmic kinetic energy plus the potential energy is equal to zero. Combining the gravitational energy equation (4) with the material energy equation (2), yields the gravitational force equation

$$
\frac{\ddot{a}}{a}=\frac{4 \pi G}{3}(2 \lambda+3 \eta-3 p-\rho) .
$$

Let us examine what we have here. Notice, first of all, that the cosmological constant does not appear in the material energy equation (2), because the density and pressure contributions exactly cancel by definition (1). On the other hand, the creativity $\eta$ could also cause the density $\rho$ to increase in (2). This in turn will cause the potential energy in (4) to decrease, thereby inducing a corresponding increase in the kinetic energy of the matter content. Thus, creativity generates an inflationary force in (5) just like $\lambda$. Also notice that $\eta$ contributes directly to the material energy (3), but not to the gravitational energy (4); whereas $\lambda$ contributes directly to the gravitational energy, but not to the material energy. Therefore, let us say, the vacuum contribution consists of two parts: a variable part $\eta$, which represents matter creation, and a constant part $\lambda$, which represents the static gravitational potential of the vacuum. We shall set the constant part aside as a separate issue, and simply assume $\lambda=0$.

For lack of a better idea, the vacuum is usually modeled with a scalar field. If $\lambda$ is not constant, if it is replaced by a cosmic scalar field with a changing, nonzero vacuum expectation value, then, obviously, that will affect the material energy equation (3), and the total amount of matter and radiation in one form or another will change. In a Robertson-Walker universe, a charged scalar field $\phi$ with potential $\Phi\left(\phi^{*} \phi\right)$ has density and total pressure given by

$$
\rho=T_{00}=\dot{\phi}^{*} \dot{\phi}+\Phi, \quad p-\eta=T_{11}=\dot{\phi}^{*} \dot{\phi}-\Phi .
$$


Alternatively, however, we could partition the total pressure into an energyconserving part $p$, and an energy-nonconserving part $\eta$, as follows:

$$
\rho=p+\eta, \quad p=\dot{\phi}^{*} \dot{\phi}, \quad \eta=\Phi .
$$

The difference is that $\eta$ acts like a pressure, whereas $\lambda$ acts as both a pressure and a density in (1). At the potential minimum, the vacuum is stable, and we have no matter creation or inflation. Away from the minimum, we have an unstable false vacuum, which acts like a cosmological constant.

Current fashion is to view the early Universe in terms of a quantum phase transition involving instantons [Coleman, DeLuccia (1980)]. However, it is doubtful that such macroscopic processes are quantumly coherent. By the identification (7), matter creation can be well described classically in terms of creativity $\eta$, and this is effectively equivalent to a quantum description in terms of a scalar field.

As far as we know, the vacuum does not have an equation of motion or a definite energy. Should it? If the energy density of the vacuum $\lambda$ equals zero for some fundamental reason, then it should be the same before and after creation. How would we determine that?

If energy were always conserved, then the total energy of the Universe should have remained constant. But it has increased, if we accept the inflationary (negative pressure) scenario. Equation (4) merely says that the cosmic kinetic energy of matter must balance the gravitational potential energy. However, the rest mass-energy must come from some non-gravitational source, characterized by $\eta$ in $(3)$.

\section{Creativity in Cosmology}

Let us model the creativity with the simple step function in Fig. 1. First, we have $\eta=\eta_{i}$ until inflation ends at $V_{i}$. The slow-roll condition corresponds to $p=0$ in (7). Then, after a long pause, dark energy appears with $\eta=\eta_{d}$, starting at $V_{d}$, and continuing unchanged until the present $V_{0}$. After recombination, we can safely assume $p=w=\lambda=k=0$, in which case, the material energy equation (3) reduces to

$$
E=\rho V=\int_{0}^{V} \eta d V=\eta_{i} V_{i}+\eta_{d}\left(V-V_{d}\right) \theta\left(V-V_{d}\right)
$$




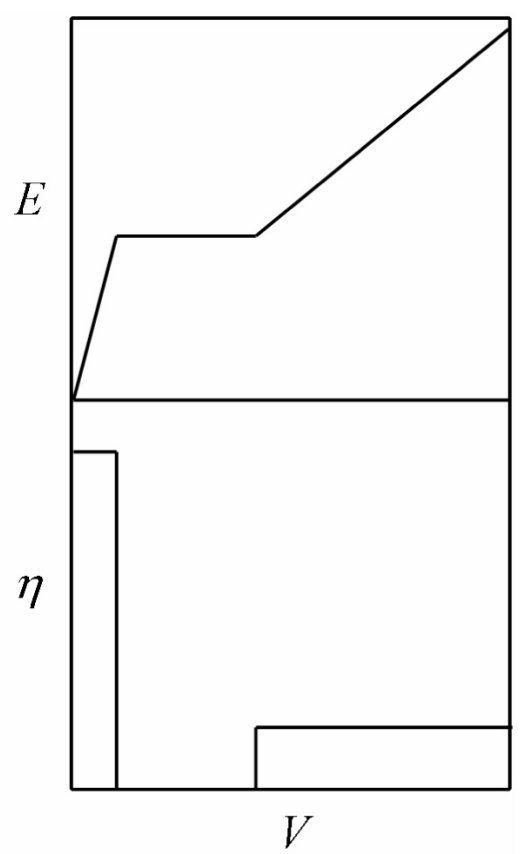

Fig. 1. Total energy $E$ and creativity $\eta$ versus the cosmic volume $V$.

For cold creation, the material pressure $p$ is zero, and the material energy $E$ is a function of the volume $V(t)$, which serves as a cosmic clock. Using (8), we can rewrite the gravitational energy equation (4) as follows:

$$
\begin{aligned}
& \frac{H^{2}}{H_{0}^{2}}=\left[\left(1-\Omega_{d}\right)(1+z)^{3}+\Omega_{d}\right][1-\theta(z-x)]+y \Omega_{d}(1+z)^{3} \theta(z-x), \\
& \frac{\dot{a}}{a}=H, \quad \frac{\eta_{i} V_{i}}{\eta_{d} V_{0}}=y, \quad \frac{V_{0}}{V}=(1+z)^{3}, \quad \frac{V_{0}}{V_{d}}=(1+x)^{3}, \\
& \frac{8 \pi G \eta_{d}}{3 H_{0}^{2}}=\Omega_{d}=\left[y-(1+x)^{-3}+1\right]^{-1} .
\end{aligned}
$$

The resulting Hubble equation (9) shows that cold creation can mimic a cosmological constant, as was previously noted by others [Lima, Alcaniz (1999)], [Zimdahl et al. (2001)], using different models for the creativity. We get no acceleration at high $z$, because we assume that creation stopped, and then started again after the earliest black holes formed. Inserting $\Omega_{d}=0.72 \pm 0.04$ [Spergel et al. (2006)] and $x>6$ [Haiman, Quartaert (2004)] into (11) gives

$$
M_{i} / M_{d} \approx y=\Omega_{d}^{-1}+(1+x)^{-3}-1=0.39 .
$$

These two mass values characterize two unrelated episodes of matter creation, separated by a period with no creation. Employing $\eta \propto V^{-1}$ over the entire range yields a logarithmic total energy, which does not fit the observations. 


\section{Vacuum Accretion}

The coincidence in the mass values could come about if ordinary matter, after it has condensed into galaxies, somehow induces the creation of more matter. That, at least, puts the two numbers into roughly the same ballpark. If the present cosmic acceleration is really caused by matter creation, then what matter is being created, and where?

One intriguing possibility is that collapsed objects or black holes may be accreting energy from the vacuum, thereby becoming heavier. For simplicity, let us suppose that the mass fraction changes as

$$
\dot{m} / m=\gamma \theta\left(\rho_{m} / \rho_{c}-1\right),
$$

where $\gamma$ is a constant characterizing the growth rate. According to this formula, vacuum accretion occurs only when the mass density $\rho_{m}$ exceeds some unknown critical density $\rho_{c}$. Based on equations (2) and (5), we can write

$$
\Lambda_{d}=8 \pi G\left(\frac{3 \dot{M}}{2 V}\right)=8 \pi G \sum_{m}\left(\frac{3 \dot{m}}{2 V}\right)=\sum_{m} \Lambda_{m}
$$

where $\Lambda_{d}$ is the effective cosmological constant due to dark energy. Thus, for a mass that changes with time, the full gravitational potential is

$$
\Phi_{\text {grav }}=-\frac{G m}{r}-\frac{1}{6} \Lambda_{m} r^{2}
$$

Since acceleration is minus the gradient, a positive $\Lambda_{m}$ gives repulsion. In practice, however, we cannot separate the long-range repulsive force due to a single mass from the repulsive forces of other masses, and only the total $\Lambda_{d}$ can be used to characterize the repulsion. Whatever the cause of the mass growth, equation (14) tells us that there must be a repulsive gravitational force proportional to the growth rate.

Strictly speaking, a black hole is defined by an event horizon: a hypothetical construct that has never been seen, and perhaps never will [Abramowicz (2002)]. There is no evidence that general relativity holds true at very high curvatures, where the horizon would emit blackbody radiation with temperature $T_{h}=1 / 8 \pi G m$. More likely, new physics would come into play, and stop the collapse before the event horizon is reached. If a collapsed object emits no light, then it could legitimately be called a near black hole [Hoyle et al. (2000)], which means that it lacks an event horizon, because there is some maximum density keeping the surface area greater than the horizon area. However, this 
cannot be true for a supermassive non-dense body that is engulfed by an event horizon long before any extreme densities are encountered. Cut off from our Universe by an event horizon, such a supermassive black hole probably can not grow by vacuum accretion.

If primordial black holes were produced in the Big Bang, then according to the evaporation theory [Hawking (1974)], some of them should be exploding right now. Maybe the reason such flashes have not been seen is that accretion always predominates over evaporation, so that all black holes must grow rather than diminish. Furthermore, it is possible that collapsed objects and black holes of various sizes could account for most of the dark matter.

Extreme conditions are most likely to be found within collapsed objects, where the potential $\Phi$ could become positive (again). Highly energetic interactions in the core could produce Higgs bosons, which would mean that at least a small amount of false vacuum is present. False vacuum gives rise to matter creation, and so the total mass of the collapsed object should grow. Exactly how fast vacuum accretion would occur is difficult to say. Outside, the only detectable manifestation of the mass growth would be a cosmic repulsion, which effectively looks like a varying $\Lambda_{d}$.

Any theory of an expanding Universe must involve matter creation in one form or another. In our theory, matter is slowly being created within collapsed objects, where it remains, simply adding to the mass. By contrast, in the Quasisteady-state Cosmology [Hoyle et al. (2000)] it is proposed that supermassive black holes grow and eventually explode. They eject newly-created ordinary matter, causing their host galaxies to grow and split into new galaxies. In this way the average matter density $\rho$ remains constant, while the Universe expands due to the repulsion generated by matter creation. Unfortunately, it may be impossible to evaluate all the possible scenarios by astronomy alone.

\section{Extreme Calorimetry}

Our proposed explanation for the present cosmic acceleration is based on the supposition that collapsed objects or black holes can interact with the vacuum. How could the vacuum remain inert under such extreme conditions? In gravitational theories based on condensed matter physics [Volovik (2003)], the interface between a black hole and the vacuum is naturally unstable. Lorentz violations, if they exist [Mattingly (2005)], would have a profound effect on cosmology. Such violations might have occurred in the Big Bang, when a particular reference frame was selected, and a huge amount of matter was suddenly created. It seems plausible that the same physical process could happen again, provided the conditions were right. 
It is commonly believed that the conditions that once prevailed in the Big Bang are reproduced in high-energy particle collisions. These are occurring all the time in the form of cosmic rays [Cronin (1999)]. Although cosmic rays are extremely powerful and cheap, we can not use them to test energy conservation, because we do not know what we are dealing with unless we assume energy is conserved in the first place. If mass-energy is created in the collision event, then the particles created will have a total energy greater than the incident particle, causing us to overestimate its power. To test energy conservation, we need to use colliding beams where the incident energy is known. If the visible particles emerging from a collision show an increase in the total energy, then that would constitute clear evidence for vacuum instability.

A serious test of energy conservation in high-energy collisions will require careful analysis of many complex multi-particle events. This will be very difficult if we are not allowed to use energy conservation. For instance: If the final energy exceeds the initial energy, it is quite possible that two separate collisions occurred at nearly the same time when the two beams containing many protons passed through the detector. The event must be rejected, even though it might be genuine. One would think that energy conservation has already been thoroughly tested by the many experiments that have been performed over the years. But they all tacitly use energy conservation to filter out spurious data, and event analyzers automatically discard nonconserving events as invalid.

The only relevant tests of energy conservation that have been done so far involve simple two-body reactions. For example, by measuring the energies of gamma rays emitted following neutron capture by atomic nuclei, it has recently been verified [Rainville et al. (2005)] that mass-energy is conserved to an accuracy of $0.00004 \%$.

But such tests are not decisive because they merely select those events where energy is likely to be conserved, not the events where many particles are created, which would be characteristic of a false vacuum. Since the vacuum looks perfectly stable at low energies, a search for direct signs of vacuum instability will require a particle accelerator. Fortunately, collisions are easy to isolate and interpret, compared to other experimental approaches.

\section{Conclusion}

According to recent observations [Riess et al. (2004)], the effective cosmological constant appears to remain unchanged out to $z=1.6$. If the effective cosmological constant were found to increase at higher red shifts, then that would clearly disprove our theory; because at earlier times, less mass was tied up in collapsed objects or black holes. Of course, proving that isolated black 
holes actually gain weight will be very difficult, and they may only be observable by gravitational lensing [Bennett (2002)]. Perhaps the only practical way to distinguish vacuum accretion from ordinary matter accretion is by the cosmic acceleration produced by the former.

Astronomical evidence is not enough. To make further progress, we need to find some direct evidence for energy nonconservation in the laboratory. Inflation theory requires the existence of a mass-creating inflaton field, or something like it, but there is yet no sign of it in high-energy collisions. Needless to say, such experiments must be carefully designed, since energy conservation cannot be used to filter out extraneous background signals.

\section{References}

[Abramowicz (2002)] Abramowicz, M. A., Kluzniak, W., Lasota, J. P., 2002. A\&A 396, L31.

[Bennett (2002)] Bennett, D. P. et al., 2002. ApJ 579, 639.

[Bronstein (1933)] Bronstein, M., 193. Phys. Z. Sowjetunion 3, 73.

[Coleman, DeLuccia (1980)] Coleman, S., DeLuccia, F., 1980. Phys. Rev. D 21, 3305 .

[Cronin (1999)] Cronin, J. W., 1999. Rev. Mod. Phys. 71, S165.

[Haiman, Quartaert (2004)] Haiman, D., Quartaert, E., 2004. The formation and evolution of the first massive black holes, in: Barger, A. J. (Ed.), Supermassive Black Holes in the Distant Universe. Kluwer, Dordrecht, pp 147-186.

[Hawking (1974)] Hawking, S. W., 1974. Nature 248, 30.

[Hoyle et al. (2000)] Hoyle, F. Burbidge, G., Narlikar, J., 2000. A Different Approach to Cosmology. Cambridge University Press, Cambridge.

[Kragh (1996)] Kragh, H., 1996. Cosmology and Controversy. Princeton University Press, Princeton.

[Lima et al. (1996)] Lima, J. A. S., Germano, A. S. M., Abramo, L. R. W., 1996. Phys, Rev. D 53, 4287.

[Lima, Alcaniz (1999)] Lima, J. A. S., Alcaniz, J .S., 1999. A\&A 348, 1.

[Mattingly (2005)] Mattingly, D., 2005. Modern tests of Lorentz invariance. Liv. Rev. Rel. 8, 5. livingreviews.org/lrr-2005-5.

[Perlmutter et al. (1999)] Perlmutter, S. et al., 1999. ApJ 517, 565.

[Prigogine et al. (1989)] Prigogine, I., Gehenian, J, Gunzig, E., Nardone, P., 1989. Gen. Rel. Grav. 21, 767. 
[Rainville et al. (2005)] Rainville, S. et al., 2005. Nature 438, 1096 (2005).

[Riess et al. (1998)] Riess, A. G. et al., 1998. AJ 116, 1009.

[Riess et al. (2004)] Riess, A. G. et al., 2004. ApJ 607, 665.

[Spergel et al. (2006)] Spergel, D. et al., 2006. Wilkinson Microwave Anisotropy Probe (WMAP) three year results: implications for cosmology. astro-ph/0603449.

[Volovik (2003)] Volovik, G. E., 2003. The Universe in a Helium Droplet. Clarendon Press, Oxford.

[Zimdahl et al. (2001)] Zimdahl, W., Schwarz, D. J., Balakin, A. B., Pavón, D., 2001. Phys. Rev. D 64, 063501. 